
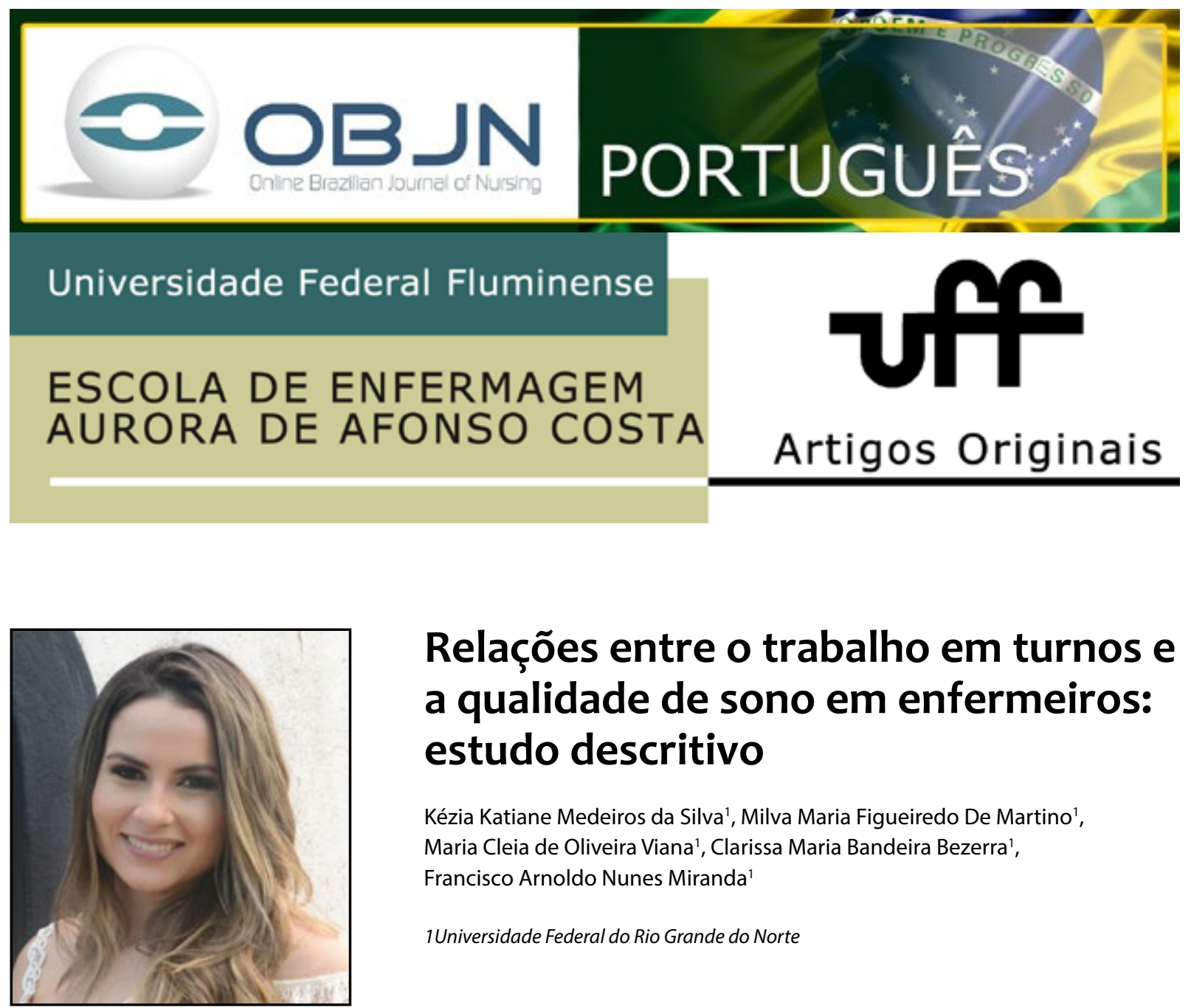

\title{
Relações entre o trabalho em turnos e a qualidade de sono em enfermeiros: estudo descritivo
}

\author{
Kézia Katiane Medeiros da Silva', Milva Maria Figueiredo De Martino', \\ Maria Cleia de Oliveira Viana', Clarissa Maria Bandeira Bezerra', \\ Francisco Arnoldo Nunes Miranda ${ }^{1}$ \\ 1 Universidade Federal do Rio Grande do Norte
}

\section{RESUMO}

Objetivo: analisar o ciclo vigília-sono e a qualidade do sono em enfermeiros que trabalham por turnos num hospital universitário. Método: delineamento do tipo transversal e descritivo, com uma abordagem quantitativa. Resultados: o ciclo vigília-sono foi avaliado por meio do diário de sono, num total de 104 enfermeiros, com idade média de 39 anos. Para medir a qualidade do sono, calculou-se o Índice Qualidade de Sono de Pittsburgh (PSQI). A percepção da qualidade do sono autorreportada pela Escala Análoga Visual (VAS) foi de 6,79 pontos, em média, para o sono do grupo diurno e 5,36 pontos para o sono do grupo noturno, com diferença estatística. No PSQI, obteve-se uma média de 7 pontos. Conclusão: os dados permitiram considerar o sono dos enfermeiros como sendo de má qualidade para o grupo do noturno. A falta de hábitos desportivos e o esquema de trabalho em turnos podem ter influenciado na qualidade do sono.

Descritores: Sono; Trabalho em Turnos; Enfermeiras; Ritmo Circadiano; Saúde do Trabalhador. 


\section{INTRODUÇÃO}

O sono, como todas as funções fisiológicas, segue um ritmo denominado circadiano, caracterizado por períodos de $24 \mathrm{~h} \pm 4 \mathrm{~h}$. Estímulos ambientais, como ciclos de claro-escuro, temperatura e, sobretudo, a presença de luz, conhecidos coletivamente como \zeitgebers", podem influenciar os ritmos circadianos ${ }^{(1-2)}$.

$A$ ausência de estímulo luminoso sobre o relógio biológico resulta em perda de sincronia entre o ritmo do sono normal de 24 horas e o ciclo claro/escuro. Quando os indivíduos são mantidos em constante escuridão e isolamento temporal, o relógio biológico reverte sua periodicidade endógena com um ritmo de livre curso, que geralmente é maior do que 24 horas ${ }^{(3)}$.

O trabalho por turnos constitui, atualmente, um problema para a maioria dos trabalhadores e um enorme desafio para a saúde ocupacional, principalmente para a equipe de enfermagem. A prática do trabalho noturno passou a ser realizada de forma contínua a partir da Revolução Industrial, em que as fábricas passaram a funcionar no período de 24 horas $^{(4)}$.

As mudanças nos horários de trabalho levam o indivíduo a apresentar o aumento da sonolência diurna, principalmente para aqueles que trabalham no turno diurno e acordam muito cedo para ir trabalhar e os do turno noturno que dormem poucas horas ou não dormem devido ao horário de trabalho(5-2).

Enfermeiros lidam constantemente com os efeitos dessas alterações no ciclo vigília-sono, que podem causar como consequências um maior risco de acidentes de trabalho e um comprometimento da qualidade de vida(5).

Pesquisas mostram que trabalhadores da enfermagem que atuam tanto no turno noturno como no diurno passam por alterações corporais e mentais após prestarem o serviço. Foram observados, em prestadores de serviço do turno noturno, pior qualidade do sono e, nos do diurno, problemas gastrointestinais e estresse $\mathrm{e}^{(5)}$.

Desse modo, este estudo teve como objetivo analisar as características do padrão de sono nos enfermeiros dos turnos diurno e noturno e relacioná-los com a qualidade do sono.

\section{MÉTODO}

Estudo observacional descritivo de corte transversal e de abordagem quantitativa.

A população da pesquisa foi composta por enfermeiros dos turnos diurno e noturno de trabalho, totalizando 104 sujeitos. Como critérios de exclusão, os indivíduos que exerciam as suas atividades há menos de seis meses no hospital foram desconsiderados juntamente aos que se encontravam em período de licença ou de férias.

$O$ regime de trabalho da equipe de enfermagem do Hospital Universitário Onofre Lopes (HOUL), da Universidade Federal do Rio Grande do Norte, consta de jornadas de trabalho de seis horas diárias para o turno diurno, com uma folga semanal, e, para o noturno, jornadas de 12 horas de trabalho por 36 horas de folga.

Os instrumentos utilizados foram: questionário para dados sociodemográficos da população, Diário de Sono, Escala Análoga Visual e Questionário Índice de Qualidade do Sono de Pittsburgh (PSQI).

$O$ desenvolvimento do estudo atendeu às normas nacionais e internacionais de ética em pesquisa envolvendo seres humanos. Todos assinaram o Termo de Consentimento Livre Esclarecido. Os dados foram coletados em sua totalidade no período de janeiro a setembro de 2015 , no qual enfermeiros anotavam diariamente observações sobre seu sono por sete dias: horário de deitar, de dormir, de acordar, latência do sono, quantidade total de horas dos sonos diurno e noturno, cochilos, qualidade do sono e 
como acordavam. Posteriormente, eles responderam ao Questionário Índice de Qualidade do Sono de Pittsburgh.

O nível de significância foi de $p<0,05$. $O$ teste não paramétrico Mann-Whitney foi utilizado para comparar os grupos de trabalho e o padrão do sono e para fazer as associações no teste Qui-Quadrado e no Teste de Correlação de Spearmann entre as variáveis do PSQI e o padrão de sono.

Os dados da pesquisa foram coletados após apreciação e aprovação do Comitê de Ética em Pesquisa da instituição sob parecer número 751.579 , sendo respeitados todos os aspectos éticos. A fim de garantir sigilo da identidade dos entrevistados, identificaram-se os sujeitos a partir da ordem de inclusão por um número arábico sequencial precedido das iniciais do nome completo.

\section{RESULTADOS}

A primeira etapa constituiu-se da caracterização da população. A amostra final foi composta por 104 enfermeiros, divididos em dois setores: hospitalar, que correspondeu a $65,40 \%$ da amostra, sendo $33,70 \%$ do turno diurno e $31,70 \%$ do turno noturno, e ambulatorial, que correspondeu a $34,60 \%$ dos enfermeiros, sendo $27,90 \%$ do turno diurno e $6,7 \%$ do turno noturno.

Houve predominância dos sujeitos do sexo feminino de uma forma geral $(n=94)$, distribuídos no turno diurno $(n=58)$ e no turno noturno $(n=36)$. Ao serem agrupados em relação à faixa etária, $73 \%$ dos sujeitos tinham entre 24 e 45 anos de idade, dentre os quais $41,30 \%$ pertenciam ao turno diurno e $31,70 \%$, do turno noturno. Observou-se que, em relação ao estado civil dos sujeitos, 52,90\% eram casados e $32,1 \%$, solteiros.
Quanto ao tempo de profissão, os enfermeiros foram distribuídos em: aqueles que tinham entre 1 e 25 anos, totalizando $80,80 \%$ da amostra, dentre os quais $48,10 \%$ eram do turno diurno e $32,70 \%$ eram do turno noturno; e os com tempo de trabalho de 26 a 48 anos, o que correspondeu a $19,30 \%$ da amostra nos dois turnos de trabalho. Do total de enfermeiros, $55,70 \%$ afirmaram ter outro emprego. Dos enfermeiros que trabalhavam em dupla jornada, 26,90\% trabalhavam no turno da manhã e $28,80 \%$, no turno da noite.

Do total dos participantes, 76,90\% faziam uso de estimulantes. Entre eles, 51,90\% eram do turno diurno e $25 \%$, do turno noturno, e o horário de preferência para ingestão era o período diurno, totalizando 43,30\%. Quanto à ingestão de medicamentos de uma forma geral, constatou-se que $51 \%$ dos sujeitos faziam uso de algum tipo de medicação, dos quais $27,90 \%$ trabalhavam no turno diurno e $23,10 \%$, no turno noturno. Quanto ao consumo de bebida alcoólica socialmente, observou-se que 52,90\% dos enfermeiros não faziam uso, no entanto a maior frequência foi para o grupo do turno diurno (35,60\%).

Entre os sujeitos participantes da pesquisa, $56,70 \%$ afirmaram não praticar atividade física. Comparando as variáveis sociodemográficas entre os turnos de trabalho, verificou-se que houve diferença estatística, constatada pelo teste Qui-Quadrado, para as seguintes variáveis: setor hospitalar e ambulatorial $(p=0,003)$ no turno diurno, possuir mais de um trabalho $(p=0,002)$ no turno noturno e usar bebida estimulante $(p=0,021)$ no turno diurno. Para os valores de médias das variáveis do padrão de sono, quando comparado o turno diurno com o noturno, notou-se diferença estatística pelo teste Mann-Whitney com valor de $(p>=0,005)$, conforme a Tabela 1. 
Tabela 1. Características do padrão de sono dos enfermeiros segundo o turno de trabalho. Natal/ RN, 2016.

\begin{tabular}{lccccc}
\hline \multirow{2}{*}{ Variáveis } & \multicolumn{2}{c}{ Diurno } & \multicolumn{2}{c}{ Noturno } & \multirow{2}{*}{ p-valor } \\
\cline { 2 - 5 } & Média & Desvio padrão & Média & Desvio padrão & \\
\hline Tempo de Cochilo (min.) & 14,00 & 15,48 & 39,16 & 47,11 & $\mathbf{0 , 0 0 3}$ \\
Latência do Sono (min.) & 27,05 & 21,29 & 36,27 & 24,12 & $\mathbf{0 , 0 1 3}$ \\
Tempo de Sono (horas) & 6,97 & 1,02 & 5,97 & 1,04 & $<0,001$ \\
Como se sentiu ao acordar (0-10) & 6,61 & 1,87 & 5,57 & 1,76 & $\mathbf{0 , 0 1 7}$ \\
Qualidade do Sono noturno (0-10) & 6,79 & 1,93 & 5,36 & 1,85 & $\mathbf{0 , 0 0 1}$ \\
\hline
\end{tabular}

*teste Mann-Whitney, valor de $p<0,05$

Fonte: própria pesquisa, 2016.

Na Tabela 2, verificou-se que, na população estudada, 39 (60,94\%) enfermeiros do turno diurno apresentavam sono de má qualidade enquanto 25 (39,06\%) apresentaram escore de boa qualidade. De acordo com a classificação obtida com o PSQI, os enfermeiros do noturno mostraram 34 (85\%) para o sono ruim e apenas seis (15\%) para o sono bom.

As análises feitas pelo teste Qui-Quadrado demonstraram diferença estatisticamente significativa ( $p=0,0091$ ) entre os turnos de trabalho e PSQI, indicando que a população de enfermeiros do noturno apresentava uma má qualidade de sono.
Dentre as variáveis estudadas, os dados mostraram que houve correlação significativa entre a qualidade do sono e as variáveis do padrão de sono: latência do sono $(p=0,010)$, total de sono $(p=0,002)$, como se sentiu ao acordar $(P<0,001)$ e qualidade do sono noturno $(\mathrm{P}<0,001)$.

\section{DISCUSSÃO}

Participaram os enfermeiros do turno diurno que trabalhavam nos horários matutino ou vespertino, nos períodos, respectivamente,

Tabela 2. Comparação do turno de trabalho em função da qualidade do sono (PSQI). Natal/RN, 2016

\begin{tabular}{|c|c|c|c|c|c|}
\hline \multirow{3}{*}{ Variável } & \multicolumn{4}{|c|}{ PSQI categorizado } & \multirow{3}{*}{ p-valor* } \\
\hline & \multicolumn{2}{|c|}{ Boa } & \multicolumn{2}{|c|}{ Ruim } & \\
\hline & $\mathbf{n}$ & $\%$ & $\mathbf{n}$ & $\%$ & \\
\hline \multicolumn{6}{|l|}{ Turno } \\
\hline Diurno & 25 & 39,06 & 39 & 60,94 & \multirow{2}{*}{0,0091} \\
\hline Noturno & 6 & 15,00 & 34 & 85,00 & \\
\hline
\end{tabular}

*p-valor obtido por meio do teste Qui-Quadrado.

Fonte: própria pesquisa, 2016.

Tabela 3. Correlação entre o escore da qualidade do sono do PSQI e as variáveis do padrão do Diário do Sono. Natal/RN, 2016

\begin{tabular}{lcccc}
\hline \multicolumn{1}{c}{ Variáveis } & Latência & Total de sono & Como se sentiu ao & $\begin{array}{c}\text { Qualidade sono } \\
\text { acordar }\end{array}$ \\
\hline Pontuação PSQI & $0,2506^{*}$ & $-0,2950^{*}$ & $-0,4564^{*}$ & $-0,4276^{*}$ \\
p-valor & $\mathbf{0 , 0 1 0 3}$ & $\mathbf{0 , 0 0 2 4}$ & $<\mathbf{0 , 0 0 0 1}$ & $<\mathbf{0 , 0 0 0 1}$ \\
\hline
\end{tabular}

*Coeficiente de correlação de Spearman

Fonte: própria pesquisa, 2016. 
das $7 \mathrm{~h}$ às $13 \mathrm{~h}$ ou das $13 \mathrm{~h}$ às $19 \mathrm{~h}$, e no noturno, no período das $19 \mathrm{~h}$ às $7 \mathrm{~h}$ do dia seguinte. No que se refere ao sexo, houve predominância do feminino. Outros estudos também encontraram a predominância de mulheres no exercício da profissão de enfermeiro. Isso pode ser explicado pelo fato de que, ainda nos dias de hoje, a mulher moderna faz sua escolha profissional influenciada pela história familiar e social, que reflete a sua socialização para exercer os papéis femininos, como no caso da enfermagem ${ }^{(6-7)}$.

Ao serem agrupados em relação à faixa etária, $73 \%$ dos sujeitos estavam na faixa dos 24 e 45 anos de idade. No entanto, outro estudo mostrou que a faixa etária dos enfermeiros era constituída, predominantemente, de adultos, entre 20 e 49 anos de idade, resultado semeIhante aos achados desta pesquisa ${ }^{(6)}$.

Observou-se, de uma forma geral, que os enfermeiros apresentaram uma qualidade do sono ruim quando medida pelo PSQI, tanto para o grupo do diurno quanto para o noturno. Alguns estudos correlacionaram o trabalho em turnos às alterações dos hábitos de sono em profissionais de enfermagem, indicando que o trabalho noturno pode prejudicar a qualidade do sono desses profissionais ${ }^{(3-6)}$.

Em uma pesquisa realizada com 1.360 enfermeiras, foi observado que aquelas que trabalhavam no turno da noite ou em turnos alternados, com prevalência da escala noturna, apresentavam um sono de má qualidade ao serem comparados àqueles que trabalhavam apenas durante o dia $^{(6-8)}$.

Outra pesquisa, realizada no norte da Noruega com 1.968 enfermeiros, mostrou uma associação entre o trabalho noturno e a má qualidade do sono. Esses dados corroboram os resultados encontrados na presente pesquisa, na qual observaram-se resultados semelhantes para ambos os turnos de trabalho do enfermeiro ${ }^{(9)}$.
Pesquisas realizadas com enfermeiros pediátricos, utilizando o PSQI, mostraram que os enfermeiros do turno da noite apresentaram uma pior qualidade do sono quando comparados aos do diurno citados, no entanto, em outros estudos ${ }^{(10-11)}$.

Quando se correlacionaram os dados da pontuação total do PSQI dos enfermeiros estudados, observou-se uma má qualidade do sono noturno com tendência significativa $(r=-0,4276$, $p=0,009)$, sendo explicado pelo ato de cochilar durante o dia.

Normalmente, os cochilos têm duração de 15 a 60 minutos, podendo ocorrer várias vezes ao dia, contudo, quando os enfermeiros aumentam a frequência ou a duração dos cochilos, podem interferir no sono noturno, como foi descrito em outro estudo, no qual os enfermeiros do turno noturno cochilavam, em média, 39,16 minutos durante o dia, gerando prejuízo à qualidade e à duração do sono durante a noite ${ }^{(12,13,14)}$.

Os resultados mostraram que o tempo total de sono dos enfermeiros do turno diurno foi maior quando se comparou com o tempo do grupo do turno noturno. Os fatores cronobiológicos e fisiológicos, como o aumento da secreção de cortisol, a redução de melatonina e o aumento da temperatura central pela manhã, não favorecem o sono dos que trabalham no período noturno, dificultando e, consequentemente, diminuindo o tempo total de sono(15).

O tempo total de sono (TTS) consistiu no período entre o horário em que o indivíduo dormiu à noite até o horário em que ele acordou na manhã seguinte. $\mathrm{O}$ adulto requer uma média de 7 a 8 horas de sono por dia. No entanto, nesta pesquisa, os enfermeiros do grupo diurno mostraram-se em média considerada normal, sendo 6,97 o tempo de sono total. Já os do noturno dormiram menos horas, resultando em uma média considerada abaixo do ideal(16).

Em uma pesquisa comparativa entre os 
grupos dos turnos diurno e noturno, ficou evidente essa diferença, concluindo que as fases do sono no diurno não são iguais ao sono no noturno(15-16). As correlações existentes entre PSQI e variáveis do diário de sono evidenciaram uma qualidade de sono ruim, com período de latência do sono maior e tempo de sono menor em comparação ao grupo do turno diurno, o qual demonstrou boa qualidade de sono(17-18).

\section{CONCLUSÃO}

Os dados permitiram considerar o sono dos enfermeiros como sendo de má qualidade no grupo do noturno, bem como mostraram alterações em todas as variáveis do padrão de sono com diferenças estatísticas. A falta de hábitos desportivos e o esquema de trabalho em turnos podem ter influenciado na qualidade do sono. Concluiu-se, então, que o trabalho noturno pode causar um impacto negativo na saúde dos trabalhadores.

\section{REFERÊNCIAS}

1. Silva KKM; De Martino MMF; Viana MCO; Bezerra CMB; Freire APCO; Silva DM; Aquino ASF. Night Shift Work and Sleep Wake Cycle in Nurses: an Integrative Review. International Archives of Medicine. 2016; 9(1): 1-9.

2. Lanzani MF, De Zavalia N, H Fontana, Sarmiento MI, Golombek D, Rosenstein RE. Alterações do ritmo de sono e parâmetros de atividade locomotora em pacientes com glaucoma avançado. Chronobiol Int. 2012; 2(9):911-919.

3. Weiblich N. Fotorreceptor e sua influência no ciclo circadiano. Revista Especialize On-line IPOG - Goiânia - 8a Edição. 2014 Dez; 9(01):1-13.

4. Silva NCD; Ferreira JVB; Albuquerque TC; Rodrigues, M. R; Medeiros MF. Transtornos á saúde mental relacionados à intensa rotina de trabalho do enfermeiro: uma revisão bibliográfica. Revista
Eletrônica Estácio Saúde. 2016; 5(2): 107-122.

5. Mendes S, De Martino MMF. Shift work: overall health state related to sleep in nursing workers. Rev Esc Enferm. USP. 2012; 46(6): 1471-1476. study. Geriatric nursing. 2016; 37 (1): 13-18.

6. Flo E; Pallesen S; MagerøyN; Moen BE; Grønli J; Nordhus IH; Bjorvatn B. Shift Work Disorder in Nurses - Assessment, Prevalence and Related Health Problems. Plos One. 2015; 7(4) 33981-33981.

7. Santos AA; Costa ORS. Qualidade de Vida no Trabalho dos Profissionais de Enfermagem que atuam no Período Noturno em um Hospital Escola do Sul de Minas Gerais. Rev Ciências em Saúde. 2015; 6(1): 43-51.

8. Santos TCMM; Faria AL; Feitosa MS; Coimbra GT; Ferreira LC; De Martino MMF. Quality and sleep disturbances of the nursing staff of a therapy unit. Journal of Nursing UFPE On Line. 2014; 8(5) 1110-1116.

9. Jensen HI; Holst JMR; Thomsen TD; Larsen JW; Eg DM; Nielsen LS. Shift work and quality of sleep; effect of working in designed dynamic light. Int Arch Occup Eviron Health. 2016; 89: 49-61.

10. Huth JJ; Eliades A; Handwork C; Englehart JL; Mensageiro J. Shift Worked, Quality of Sleep, and Elevated Body Mass Index in Pediatric Nurses. Journal of Pediatric Nursing. 2013; 28(6) 64-73.

11. 11. Shao M; Chou Y; Yeh M; Tzeng W. Sleep quality and quality of life in female shift-working nurses. Journal of Advanced Nursing. 2015; 66(7) 15651572.

12. Costa AS; Rotenberg L; Griep RH; Fischer FM. Cochilos durante o trabalho noturno em equipes de enfermagem: possíveis benefícios à saúde dos trabalhadores. Escola Anna Nery. 2015; 19(1): 33-39.

13. Xavier KGS; Vaghetti HH. Aspectos cronobiológicos do sono de enfermeiras de um hospital universitário. Revista Brasileira de Enfermagem. 2012; 65(1): 135-140.

14. Silva-Costa A; Rotenberg L; Griep RH; Fischer FM. Cochilos durante o trabalho noturno em equipes de enfermagem: possíveis benefícios à saúde dos trabalhadores. Escola Anna Nery Revista de Enfermagem. 2015; 19(1): 33-39.

15. Silva RM; Zeitoune RCG; Beck CLC; Loro MM. Matutino, vespertino ou indiferente? Produção do conhecimento sobre o cronotipo na en- 
fermagem. Revista de Enfermagem da UFSM. 2015; 4(4): 835-843.

16. De Martino MMF; Abreu ACB; Barbosa MSF; Teixeira JEM. Relação entre trabalho por turnos e padrões de sono em enfermeiros. Saúde Coletiva. 2013; 18(3) 763-768.

17. Souza SBC; Tavares JP; Macedo ABT; Moreira PW; Lautert L. Influência do turno de trabalho e cronotipo na qualidade de vida dos trabalhadores de enfermagem. Revista Gaúcha de Enfermagem. 2012: 33(4): 79-85.

18. Braga L;Torres L; Ferreira V. Condições de trabalho e fazer em enfermagem. Revista de Enfermagem.2015; 1(1): 55-63.
Todos os autores participaram das fases dessa publicação em uma ou mais etapas a seguir, de acordo com as recomendações do International Committe of Medical Journal Editors (ICMJE, 2013): (a) participação substancial na concepção ou confecção do manuscrito ou da coleta, análise ou interpretação dos dados; (b) elaboração do trabalho ou realização de revisão crítica do conteúdo intelectual; (c) aprovação da versão submetida. Todos os autores declaram para os devidos fins que são de suas responsabilidades o conteúdo relacionado a todos os aspectos do manuscrito submetido ao OBJN. Garantem que as questões relacionadas com a exatidão ou integridade de qualquer parte do artigo foram devidamente investigadas e resolvidas. Eximindo, portanto o OBJN de qualquer participação solidária em eventuais imbróglios sobre a matéria em apreço. Todos os autores declaram que não possuem conflito de interesses, seja de ordem financeira ou de relacionamento, que influencie a redação e/ou interpretação dos achados. Essa declaração foi assinada digitalmente por todos os autores conforme recomendação do ICMJE, cujo modelo está disponível em http://www. objnursing.uff.br/normas/DUDE_final_13-06-2013.pdf

Recebido: $13 / 05 / 2016$

Revisado: 26/01/2017

Aprovado: 26/01/2017 